\title{
SÍNTESE DIRETA DA PENEIRA MOLECULAR MESOPOROSA AI-SBA-15
}

\author{
E. R. ZANATTA ${ }^{1}$, L. S. da SILVA ${ }^{2}$, R. Z. RAIA ${ }^{2}$, S. M. P. MARCUCCI ${ }^{2}$, P. A. \\ ARROYO ${ }^{2}$ \\ ${ }^{1}$ Universidade Tecnológica Federal do Paraná, Núcleo de Alimentos \\ ${ }^{2}$ Universidade Estadual de Maringá, Departamento de Engenharia Química \\ E-mail para contato: elcianezanatta@utfpr.edu.br
}

\begin{abstract}
RESUMO: Existem apenas cinco procedimentos de síntese direta de peneira moleculare mesoporosa Al-SBA-15 incorporação de alumínio descritas na literatura. Neste trabalho Al-SBA-15 foi sintetizada baseada em um procedimento chamado de "ajuste de $\mathrm{pH}$ " e também foi sintetizada com uma modificação neste procedimento para reduzir o tempo total da síntese em 64 h. Foi usado como fonte de sílica o tetraetilortossilicato, de alumínio o isopropóxido de alumínio, como agente direcionador de estrutura o copolímero tribloco Pluronic P123 e solução de ácido clorídrico. A caracterização das amostras de Al-SBA-15 foram realizadas por Medidas de Fisissorção de $\mathrm{N}_{2}$, Dessorção de Amônia a Temperatura Programada (DTP/NH $\mathrm{NH}_{3}$, Difratometria de Raios X (DRX) e Espectrometria de Absorção Atômica (AA). Os resultados mostraram que o procedimento adaptado pode ser utilizado para produção da Al-SBA-15 com boa ordenação estrutural, alta acessibilidade devido ao diâmetro médio de poros obtido e quantidade de sítios ácidos moderada, além de ser produzida em menor tempo de síntese.
\end{abstract}

\section{INTRODUÇÃO}

Em 1998, pesquisadores da Universidade da Califórnia - Santa Bárbara, EUA, relataram a síntese de uma nova família de materiais mesoporosos, com organização hexagonal, a SBA-15 (sigla do nome em inglês: Santa Barbara Amorphous number 15), que expande ainda mais os canais destes materiais para até $300 \AA$ (Zhao et al., 1998). O sólido SBA-15 é um material sintetizado na forma silícica. Portanto, são de uso limitado para aplicações catalíticas por não apresentarem sítios superficiais reativos, os quais podem ser gerados pela introdução de heteroátomo na estrutura (Corma et al., 1994). Muitos esforços têm sido realizados para incorporar heteroátomo via síntese direta na SBA-15 no sentido de 
superar a limitação da baixa força ácida. Existem poucos procedimentos de síntese direta de incorporação de alumínio descritos na literatura.

Na primeira síntese direta de Al-SBA-15 em condições ácidas, foi obtida uma atividade catalítica de craqueamento de cumeno $\left(\mathrm{C}_{9} \mathrm{H}_{12}\right)$ mais elevada do que para a Al-MCM-41 (Yue et al., 1999), despertando o interesse para a atividade catalítica. Na introdução de alumínio e titânio em SBA-15, preparada em meio fortemente ácido, utilizando o método de "ajuste de pH" os resultados obtidos mostraram que um material mesoestruturado altamente ordenado com alto conteúdo de heteroátomos e razão de sílica de até 2 pode ser sintetizado (Wu et al., 2004). Assim, comprovou-se a possibilidade da incorporação de heteroátomo na estrutura. Em 2004 foi relatada uma síntese direta de Al-SBA-15 por meio da hidrólise controlada na presença de flúor (Li et al., 2004). Os resultados mostraram que o ajuste do $\mathrm{pH}$ do gel de síntese é um método eficiente para evitar a lixiviação das espécies de alumínio da estrutura. Ainda em 2004, foi descrita uma série de sínteses de Al-SBA-15 simplesmente ajustando a concentração de ácido clorídrico no gel de síntese. Por meio deste método, Al-SBA-15 com razão Si/Al inferior a 7 pôde ser preparada com sucesso apenas controlando a razão $\mathrm{H}_{2} \mathrm{O} / \mathrm{HCl}$ em 276 (Vinu et al., 2004). Em 2011, foi relatada uma síntese direta de Al-SBA-15 sem adição de ácidos (Lin et al., 2011), em que a área específica, o volume de poros e o tamanho dos mesoporos puderam ser modificados pelo simples ajuste dos parâmetros de síntese, tais como a razão molar $\mathrm{Si} / \mathrm{Al}$, cuja ordenação da estrutura melhora com a diminuição desta razão de 100 para 5, a razão molar P123/Si no gel de síntese, em que o aumento desta razão se tem um aumento no diâmetro e volume dos poros da Al-SBA-15, e a temperatura de envelhecimento, cujo aumento para $170{ }^{\circ} \mathrm{C}$ provoca o alargamento dos poros.

Portanto, o presente trabalho teve como objetivo estudar a síntese de peneira molecular mesoporosa Al-SBA-15 pelo procedimento chamado de "ajuste de $\mathrm{pH}$ " (Wu et al., 2004) e, também, por uma modificação deste procedimento, visando à redução do tempo total da síntese.

\section{SEÇÃO EXPERIMENTAL}

Procedimento 01 - Este procedimento foi denominado "ajuste pH" e foi baseado no trabalho de Wu et al. (2004). Assim, em um recipiente de teflon com tampa foi dissolvido o Pluronic P123 em $125 \mathrm{~mL}$ de solução de ácido clorídrico $\left(2 \mathrm{~mol} . \mathrm{L}^{-1}\right)$, por 12 horas, a temperatura ambiente. Após, a temperatura foi elevada para $40{ }^{\circ} \mathrm{C}$ por 4 horas e, então, adicionou-se TEOS e agitou-se por mais 4 horas. Adicionou-se o isopropóxido de alumínio e agitou-se por 20 horas. A suspensão foi transferida para autoclaves de teflon revestidas de inox, cujo enchimento foi de $2 / 3$ do volume. As autoclaves foram mantidas em estufa a $100{ }^{\circ} \mathrm{C}$, por 48 horas. As autoclaves foram retiradas da estufa e foram resfriadas, sendo a solução transferida para um béquer de teflon, em que foi corrigido o $\mathrm{pH}$ da solução para 7,5 com hidróxido de amônio $\left(\mathrm{NH}_{4} \mathrm{OH}\right)$ concentrado. A suspensão foi colocada novamente nas 


\section{9 a 22 de outubro de 2014 \\ Florianópolis/SC}

autoclaves de inox, por mais 48 horas, a $100{ }^{\circ} \mathrm{C}$. Em seguida, o sólido obtido foi filtrado, lavado com $1000 \mathrm{~mL}$ de água desionizada e seca em estufa a $100{ }^{\circ} \mathrm{C}$, por uma noite. A amostra obtida foi triturada em gral e pistilo e calcinada em ar, a $500{ }^{\circ} \mathrm{C}$, com taxa de aquecimento de $5^{\circ} \mathrm{C} \cdot \mathrm{min}^{-1}$, por 6 horas. O tempo total de síntese foi de $156 \mathrm{~h}$.

Procedimento 02 - Este tomou como base o procedimento 01, que foi adaptado para reduzir o tempo total de síntese. Deste modo, preparou-se primeiramente a solução 01, em que um recipiente de teflon com tampa foi utilizado para dissolver o Pluronic P123 em $100 \mathrm{~mL}$ de ácido clorídrico $\left(2 \mathrm{~mol} . \mathrm{L}^{-1}\right)$, durante 4 horas, a temperatura de $40{ }^{\circ} \mathrm{C}$. Uma solução 02 foi preparada em um béquer de teflon, no qual foram adicionados o TEOS, o isopropóxido de alumínio e $10 \mathrm{~mL}$ de ácido clorídrico $\left(2 \mathrm{~mol} . \mathrm{L}^{-1}\right)$, deixando sob agitação constante por 4 horas, a temperatura ambiente. Após, adicionou-se a solução 02 gota a gota na solução 01 e agitou-se por 20 horas, a $40^{\circ} \mathrm{C}$. Após as 20 horas de agitação, o pH foi corrigido para 7,5 com hidróxido de amônio $\left(\mathrm{NH}_{4} \mathrm{OH}\right)$ concentrado. A suspensão foi transferida para autoclaves de teflon revestidas de inox, cujo enchimento foi de $2 / 3$ do volume. As autoclaves foram mantidas em estufa a $100{ }^{\circ} \mathrm{C}$, por 48 horas. O sólido obtido foi filtrado, lavado com $1000 \mathrm{~mL}$ de água desionizada e seco em estufa a $100{ }^{\circ} \mathrm{C}$, por uma noite. A amostra obtida foi triturada em gral e pistilo e calcinada em ar, a $500{ }^{\circ} \mathrm{C}$, com taxa de aquecimento de $5{ }^{\circ} \mathrm{C} \cdot \mathrm{min}^{-1}$, por 6 horas. O tempo total da síntese foi de $92 \mathrm{~h}$, ou seja, $41 \%$ menor do que o utilizado para o procedimento 01 .

As amostras de Al-SBA-15 obtidas foram caracterizadas por fisissorção de $\mathrm{N}_{2}$, utilizando-se um equipamento ASAP 2020 da Micrometrics, dessorção de amônia a temperatura programada $\left(\mathrm{DTP} / \mathrm{NH}_{3}\right)$, utilizando-se um equipamento ChemBet 3000 da Quantachrome, difração de raios X (DRX), utilizando-se um equipamento Shimadzu XRD6000, espectrometria de absorção atômica (AA), utilizando-se o equipamento Varian 50B, com o objetivo de determinar as propriedades físico-químicas dos sólidos mesoporosos sintetizados pelos dois procedimentos estudados.

\section{RESULTADOS E DISCUSSÃO}

Os padrões de difração de raios $X$ das amostras calcinadas de Al-SBA-15 são apresentados na Figura 1. A identificação da fase mesoporosa ordenada ocorre quando se observa no difratograma de raios $\mathrm{X}$ pelo menos três picos de difração referentes aos planos cristalográficos (100), (110), (200) e, também, em alguns casos, o plano (210), indicativo de simetria hexagonal e elevado grau de organização (ZHAO et al., 1998). Verifica-se na Figura 1 que, embora a posição dos picos de difração possa sofrer pequenas alterações dependendo da amostra, todos os materiais sintetizados apresentaram os três picos principais, característicos da estrutura hexagonal, bem resolvidos. Como os materiais não são cristalinos em nível atômico, nenhuma reflexão a altos ângulos foi observada. Isso indica que todos os planos materiais são caracterizados por uma simetria hexagonal P6mm, típico da estrutura 
mesoporosa SBA-15. Assim, os dados obtidos no presente trabalho estão de acordo com os relatados nos artigos originais de WU et al. (2004) e YUE et al. (1999).
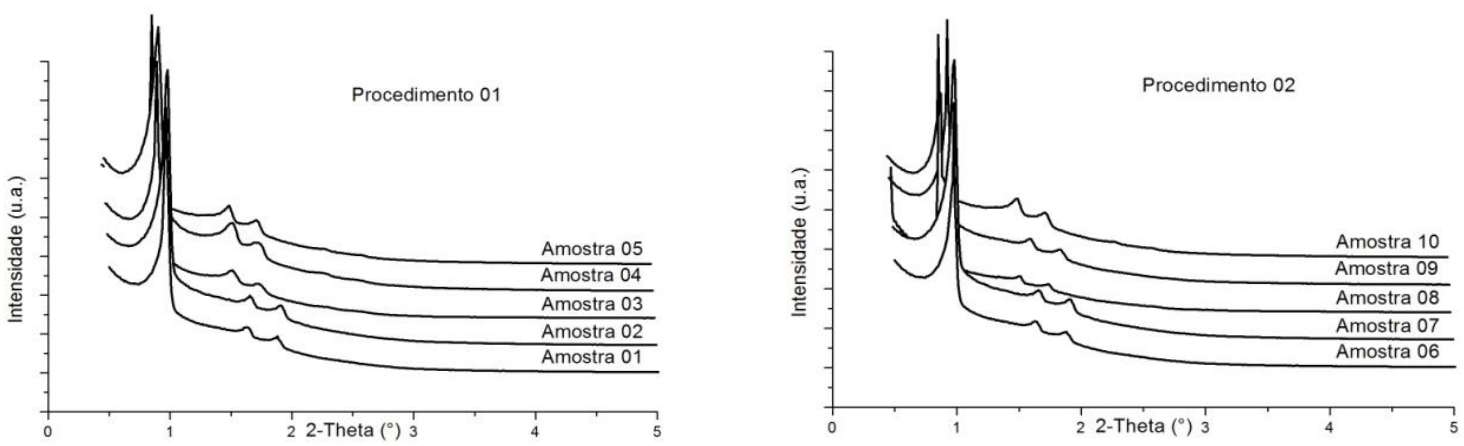

Figura 1. Difratogramas de raios $\mathrm{X}$ das amostras de Al-SBA-15 sintetizadas via procedimento 01 e 02 .

Na Tabela 1 são apresentados os resultados de composição química, tamanho da célula unitária, características texturais e acidez das amostras de Al-SBA-15 sintetizadas via procedimentos 01 e 02 .

Tabela 1 - Composição química, tamanho da célula unitária, características texturais e acidez das amostras de Al-SBA-15 sintetizadas via procedimento 01 e 02.

\begin{tabular}{|c|c|c|c|c|c|c|c|}
\hline \multirow{3}{*}{ Procedimento } & Amostra & $\begin{array}{c}\text { Razão } \\
\text { Molar } \\
\text { Si/Al }\end{array}$ & $\begin{array}{c}\mathrm{a}_{0} * \\
(\mathrm{~nm})\end{array}$ & $\begin{array}{c}\text { Área } \\
\text { Específica } \\
\text { BET } \\
\left(\mathrm{m}^{2} \cdot \mathrm{g}^{-1}\right)\end{array}$ & $\begin{array}{c}\text { Volume } \\
\text { de Poros } \\
\mathrm{BJH} \\
\left(\mathrm{cm}^{3} \cdot \mathrm{g}^{-1}\right)\end{array}$ & $\begin{array}{c}\text { Diâmetro } \\
\text { Médio de } \\
\text { Poros BJH } \\
(\AA)\end{array}$ & $\begin{array}{c}\text { Acidez } \\
\left(\mu \mathrm{mol.g}^{-1}\right)\end{array}$ \\
\hline \multirow{5}{*}{01} & 01 & 15 & 10,5 & 427 & 0,86 & 84,0 & 556 \\
\cline { 2 - 8 } & 02 & 14 & 10,4 & 477 & 0,96 & 79,0 & 484 \\
\cline { 2 - 8 } & 03 & 16 & 10,2 & 339 & 0,86 & 82,9 & 448 \\
\cline { 2 - 8 } & 04 & 15 & 10,5 & 335 & 0,79 & 78,6 & 425 \\
\hline \multirow{5}{*}{02} & 05 & 14 & 10,6 & 384 & 0,86 & 77,8 & 448 \\
\cline { 2 - 8 } & 06 & 16 & 10,2 & 338 & 0,89 & 101,7 & 423 \\
\cline { 2 - 8 } & 07 & 14 & 10,2 & 254 & 0,60 & 90,7 & 363 \\
\cline { 2 - 8 } & 09 & 15 & 10,5 & 385 & 1,07 & 109,9 & 321 \\
\cline { 2 - 8 } & 09 & 14 & 10,5 & 376 & 1,05 & 110,7 & 404 \\
\hline
\end{tabular}

*parâmetro de cela unitária $\left(a_{0}\right)$ calculado por $a_{0}=2 d_{100} / \sqrt{3}$, referente apenas ao plano 100. 
O parâmetro de rede $\left(\mathrm{a}_{\mathrm{o}}\right)$, ou parâmetro de cela unitária, que representa a soma do diâmetro médio de poro do sólido e a espessura media da parede de sílica (Wt), foi obtido a partir do valor da distância interplanar no plano (100). Pode ser observado que todos os valores obtidos para o parâmetro de cela unitária ficaram em torno de $10,5 \mathrm{~nm}$. Estes valores são muito próximos aos relatados por Lin et al. (2011), que obtiveram valores de 11-12 nm, para o mesmo tipo de sólido. É importante notar que os diferentes procedimentos de síntese estudados neste trabalho têm pouca ou nenhuma influência sobre o parâmetro de cela unitária, que foi calculado sobre o primeiro pico de reflexão, embora a intensidade dos picos possa ter uma pequena variação de um procedimento para o outro.

Pode-se observar na Tabela 1 que a quantidade de alumínio presente nas amostras sintetizadas foi acompanhada por uma queda na área específica BET. Este fenômeno pode ser atribuído à perda gradual de ordenação da estrutura mesoporosa, devido à incorporação de Al, o que também pode ser confirmado a partir das características da isoterma de fisissorção de $\mathrm{N}_{2}$. Este resultado também está de acordo com o que foi relatado por Lin et al. (2011), indicando que a qualidade da estrutura aumenta com a diminuição da razão molar Si/Al. No entanto, os resultados obtidos mostram que todas as amostras sintetizadas apresentaram áreas específicas elevadas e volumes de poros e razão $\mathrm{Si} / \mathrm{Al}$ adequadas para aplicação em processos catalíticos de hidroisomerização de alcanos (FERREIRA et al., 2009).

Os valores de acidez total apresentados na Tabela 1, para todas as amostras de AlSBA-15 sintetizadas, são próximos aos valores obtidos por Gallo et al. (2010). Estes autores obtiveram acidez de $600 \mu \mathrm{mol} \mathrm{NH} \mathrm{N}_{3} \mathrm{~g}^{-1}$, para amostras de Al-SBA-15 com razão Si/Al de 20,6, sintetizadas também pelo "método de ajuste de pH". Assim, os sólidos apresentaram mesoestrutura ordenada e acidez moderada. Esta acidez moderada dos materiais de Al-SBA-15 são desejáveis para as reações de HIDW (FERREIRA et al., 2009, LI et al., 2012).

Na Figura 2 são apresentadas as isotermas de fisissorção de nitrogênio para as amostras de Al-SBA-15 que foram sintetizadas. Pode-se verificar que as amostras sintetizadas pelo procedimento 01, amostras 01-05, apresentam isotermas do tipo IV típicas de materiais mesoporosos e histereses do tipo $\mathrm{H} 1$, características de materiais com poros regulares em formato poliédrico e com as extremidades abertas (GREGG \& SING, 1982).

Por outro lado, as amostras sintetizadas via procedimento 02, amostras 06-10, apresentam ainda isotermas do tipo IV, mas com ciclos de histerese do tipo H4, característicos de materiais com poros estreitos em fenda (GREGG \& SING, 1982). Isto indica uma alteração na porosidade secundária. No entanto, houve manutenção da área específica, volume de poros e diámetro médio de poros para o procedimento 02 , mostrando que a adaptação realizada no método 01 não teve influência significativa na estrutura final do sólido mesoporoso obtido. 

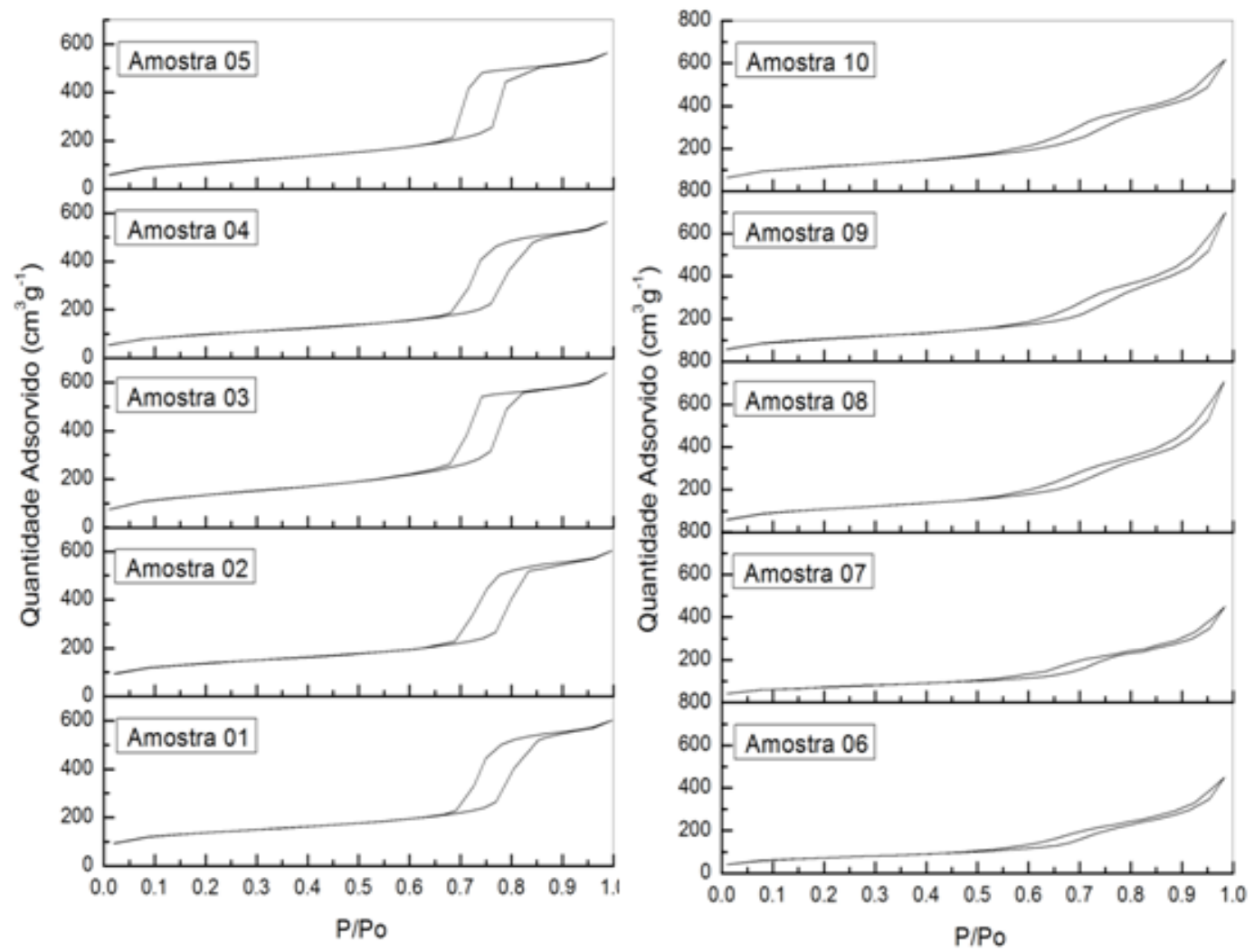

Figura 2. Isotermas de fisissorção de $\mathrm{N}_{2}$ das amostras de Al-SBA-15 sintetizadas via procedimento 01 , amostras $01-05$, e procedimento 02 , amostras 06-10.

Lin et al. (2011) encontraram uma área específica BET de $945 \mathrm{~m}^{2} \cdot \mathrm{g}^{-1}$, volume de poros de $1,03 \mathrm{~cm}^{3} \cdot \mathrm{g}^{-1}$ e tamanho do poro de $78,70 \AA$, para uma razão $\mathrm{Si} / \mathrm{Al}$ de 5 no gel de síntese e de 34 na amostra final calcinada. Embora para todas as amostras sintetizadas neste trabalho o volume de poros tenha sido um pouco menor, devido à maior incorporação de alumínio na estrutura mesoporosa, conforme os valores apresentados na Tabela 1, o diâmetro médio de poros foi maior para as amostras sintetizadas, indicando, assim, características texturais adequadas para a aplicação em catálise. Também foi possível observar que as adaptações no método de síntese não alteraram significativamente as propriedades estruturais das amostras de Al-SBA-15 obtidas.

\section{CONCLUSÕES}

A modificação na rota de síntese proposta neste trabalho apresenta potencial de aplicação para a obtenção da peneira molecular mesoporosa Al-SBA-15, por apresentar 
estrutura mesoporosa com bom grau de ordenação, boa acessibilidade, devido ao tamanho médio de poros obtido, e quantidade de sítios ácidos moderada, além de ser obtida em menor tempo de síntese.

\section{REFERÊNCIAS}

CORMA, A., FORNES, V., NAVARRO, M., PÉREZ-PARIENTE, J., "Acidity and stability of MCM-41 crystalline aluminosilicates”. Journal of Catalysis, Vol. 148, p. 569 - 574, 1994.

FERREIRA, J. F. M., SILVA, T. R., SANTOS, A. C. B., ARROYO, P. A., "Peneira molecular Al-SBA-15: Síntese, caracterização e avaliação frente à reação de hidroisomerização/hidrocraqueamento de n-hexadecano", Anais do $15^{\circ}$ Congresso Brasileiro de Catálise e $5^{\circ}$ Congresso de Catálise do Mercosul, 8p., 2009.

GAllO, J. M. R., BISIO, C., GATTI, G., MARCHESE, L., PASTORE, H., "Physicochemical characterization and surface acid properties of mesoporous [Al]-SBA-15 obtained by direct sybthesis". Langmuir, Vol. 26(8), p. 5791-5800, 2010.

GREGG, S. J., SING, K, S. W., Adsortion, surface área and porosity. $2^{\mathrm{a}}$ ed, Cap. 1-4, Academic Press., 1982.

LI, Y., ZHANG, W., ZHANG, L., YANG, Q., WEI, Z., FENG, Z., LI, C., "Direct synthesis of Al-SBA-15 mesoporous materials via hydrolysis-controlled approach". J. Phys. Chem. B, Vol. 108, p. 9739 - 9744, 2004.

LI, Y., PAN, D., YU, C., YU, F., BAO, X., "Synthesis and hydrodesulfurization properties of NiW catalyst supported on high-aluminum-content, highly ordered, and hydrothermally stable Al-SBA-15”. Journal of Catalysis, Vol. 286, p. 124 - 136, 2012.

LIN, S., SHI, L., CARROT, M. M. L. R., CARROT, P. J. M., ROCHA, J. LI, M. R., ZOU, X. D., "Direct synthesis without addition of acid of Al-SBA-15 with controllable porosity and high hydrothermal stability". Microporous and Mesoporous Materials, Vol. 142, p. $526-534,2011$.

VINU, A., MURUGESAN, V., BOHLMANN, W., HARTMANN, M., “An optimized procedure for the synthesis of AlSBA-15 with large pore diameter and high aluminum content". J. Phys. Chem. B, Vol. 108, p. 11496-11505, 2004.

WU, S., HAN, Y., ZOU, Y. C., SONG, J. W., ZHAO, L., DI, Y., LIU, S. Z., XIAO, F. S., "Synthesis of heteroatom substituted SBA-15 by the "ph-adjusting" method". Chem. Mater., Vol. 16, p. 486 - 492, 2004. 
YUE, Y., GÉDÉON, A., BONARDET, J. L., MELOSH, N., D’ESPINOSEA, J. B., FRAISSARDA, J., "Direct synthesis of AISBA mesoporous molecular sieves: characterization and catalytic activities". J. Chem. Commun, Vol. 09, p. 1967 - 1968, 1999.

ZHAO, D., FENG, J., HUO, Q., MELOSH, N., FREDRICKSON, G. H., CHMELKA, B. F., STUCKY, G. D., "Triblock copolymer syntheses of mesoporous silica". Science, Vol. 279 , p. $548-552,1998$. 